\title{
Diagnóstico y tratamiento de la perforación de colon durante la colonoscopia
}

\author{
Eduardo G arcía ${ }^{1}$, Francisco López-Köstner ${ }^{3}$, \\ Antonio Rollan', Rodrigo Muñoz ${ }^{1}$, María José Contardoa, \\ Felipe Bellolio ${ }^{1}$, Ximena G arcía ${ }^{2 b}$. \\ Diagnosis and management \\ of colon perforation after colonoscopy
}

Background: Colon perforation is an uncommon but feared complication of colonoscopy. The treatment is usually surgical but occasionally it does not require an operation. Aim: To report our experience in the diagnosis and management of colon perforation after colonoscopy. Material and methods: Retrospective review of the database of 11,720 colonoscopies. The medical records of those patients that had a perforation were reviewed. Results: Twelve perforations in patients aged 26 to 92 years (six women), were identified with a global perforation rate of $0.1 \%$. Five occurred during diagnostic and seven during therapeutic procedures. All perforations were confirmed by a plain X ray or CT scan of the abdomen. Four patients, without signs of initial diffuse peritoneal irritation, were medically treated. One of these, finally required surgery. Among operated patients, a primary suture was done in five, a primary excision without colostomy in three and a Hartmann procedure due to a severe peritoneal contamination in one. No patient died. Conclusions: There is a higher risk of colon perforation during therapeutic colonoscopies. Selected cases may be safely treated without surgery (Rev Méd Chile 2008; 136: 310-16).

(Key words: Colonoscopy; Colostomy; Intestinal perforation)

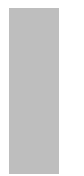

Recibido el 19 de diciembre, 2006. Aceptado el 28 de septiembre, 2007.

Departamentos de ${ }^{1}$ Cirugía Digestiva y de ${ }^{2}$ Gastroenterología, Pontificia Universidad Católica de Chile. ${ }^{3}$ Unidad de Coloproctología, Clínica Las Condes.

aAlumna Medicina, Facultad de Medicina, Pontificia Universidad Católica de Chile

${ }^{b}$ Enfermera Unidad de Endoscopia

$\mathrm{E}$ n la actualidad, la colonoscopia constituye uno de los principales métodos de diagnóstico y tratamiento de pacientes con patología colorrectal. Al ser un examen invasivo, presenta en forma inevitable complicaciones asociadas, dentro de las

Correspondencia a: Dr. Eduardo García Muñoz. Marcoleta 352, Patio Interior, Edificio División de Cirugía, Pontificia Universidad Católica de Chile. Fonos: 3543222-3543221. E mail: eduagon1972@yahoo.es cuales la perforación de colon, aunque poco frecuente, es la más grave, afectando de manera significativa la morbilidad y mortalidad de los pacientes.

$\mathrm{Su}$ incidencia se comunica en diferentes series con cifras de $0,01 \%$ a $0,4 \%{ }^{1-3}$. Esta complicación se presenta con mayor frecuencia en procedimientos terapéuticos que en diagnósticos ${ }^{4,5}$. Se han mencionado como factores de riesgo, la polipectomía con diatermia, el antecedente de cirugía abdominal previa, pacientes de edad avan- 
zada y la enfermedad diverticular sintomática asociada a deformación de colon 6,7 .

En general, el tratamiento de la perforación es quirúrgico, lo que se acompaña de morbimortalidad; no obstante, en ciertas circunstancias, también puede ser conservador con resultados exitosos $3,8,9$.

En este estudio, se analizó la experiencia en el diagnóstico y tratamiento de la perforación de colon ocurrida durante la colonoscopia.

\section{Pacientes y MÉTOdos}

Pacientes. Estudio retrospectivo realizado en base a los datos obtenidos del registro prospectivo de la morbilidad derivada de los procedimientos endoscópicos, a cargo de la enfermera coordinadora (XG), implementado en 2001. Entre enero de 2001 y junio de 2006 se realizaron 11.720 colonoscopias en la Unidad de Endoscopia del Hospital Clínico de la Pontificia Universidad Católica de Chile. Del registro de morbilidad, se obtuvo los datos de todos los pacientes con perforación derivada de una colonoscopia y se analizaron sus fichas clínicas, consignando los siguientes parámetros: intervalo entre el procedimiento y el diagnóstico, la presentación clínica, la conducta terapéutica médica o quirúrgica, el tipo de intervención quirúrgica, los hallazgos operatorios y la evolución postoperatoria. Correlacionamos la descripción del procedimiento endoscópico con los hallazgos quirúrgicos para inferir el mecanismo etiológico.

Procedimiento. En la Unidad de Endoscopia, los procedimientos son realizados por gastroenterólogos y cirujanos digestivos acreditados. Los subespecialistas en entrenamiento son supervisados directamente por los médicos acreditados. Todo paciente al que se le realiza un procedimiento endoscópico se le advierte los potenciales riesgos, debiendo firmar un protocolo de consentimiento informado previo al examen. La preparación de colon de preferencia es el Fleet fosfosoda ${ }^{\circledR}$ y a todos los pacientes se les ofrece sedación (midazolam-meperidina). En casos seleccionados se realiza el procedimiento endoscópico bajo anestesia general. Los procedimientos son realizados con videoendoscopios flexibles marca Fujinon ${ }^{\circledR}$ (modelos EC-450HL5, EC-450DL5,EC-490ZW5/L) y
Olympus ${ }^{\circledR}$ (modelos CFVL 2011375, CFVL 2111655, CFVL 2212012).

\section{Resultados}

En un periodo de 6 años se realizaron 11.720 colonoscopias, diagnosticándose 12 perforaciones (tasa de $0,1 \%$ global). La edad promedio de los pacientes complicados fue de 69 años (26-92), 6 de ellos mujeres.

Del total de colonoscopias, 8.790 fueron procedimientos diagnósticos donde ocurrieron 5 perforaciones (incidencia 0,056\%) y en 2.930 colonoscopias terapéuticas ocurrieron 7 perforaciones (incidencia 0,23\%).

El diagnóstico se sospechó durante las primeras $6 \mathrm{~h}$ en 7 pacientes, entre las 6 y $24 \mathrm{~h}$ en $4 \mathrm{y}$ sólo en uno después de las 36 h de evolución. En todos los pacientes se confirmó la perforación mediante la visualización de aire subdiafragmático (radiografía simple de abdomen de pie/tomografía computada de abdomen).

Se observaron factores de riesgo descritos para perforación en 10 de los 12 pacientes, los que se presentan en la Tabla 1.

En cuatro pacientes con dolor abdominal y distensión, sin signos de irritación peritoneal difusos, se optó por un tratamiento médico, consistente en ayuno y antibióticos endovenosos (Cefotaxima $1 \mathrm{gr}$ c/8 h iv - Metronidazol 500 mg c/8 h iv). Sólo uno de ellos evolucionó con signos de irritación peritoneal difusos después de las 8 h de observación, por lo que fue intervenido quirúrgicamente. Los otros tres fueron dados de alta en buenas condiciones generales sin haber mediado una operación.

Del total de 9 pacientes intervenidos, en 8 no había evidencia de contaminación peritoneal masiva y, sólo se encontraron lesiones lineales menores $(<2 \mathrm{~cm}), 5$ de ellos fueron tratados con sutura primaria. A tres pacientes se les realizó una resección segmentaria, uno por la presencia de un tumor asociado y dos por presentar desgarros murales extensos $(>3 \mathrm{~cm})$. Una paciente reingresó a las $36 \mathrm{~h}$ postcolonoscopia con signos de irritación peritoneal y sepsis, por lo que se le practicó una laparotomía de urgencia, cuyo hallazgo fue una perforación lineal $>2 \mathrm{cms}$ con gran contaminación peritoneal, se realizó aseo peritoneal y una operación de Hartmann (Figura 1). 
Tabla 1. Tipo de colonoscopia y factor asociado a perforación

\begin{tabular}{|llll|}
\hline Paciente & Procedimiento & \multicolumn{1}{c|}{ Factores de riesgo } & Edad (años) \\
\hline Masculino & Diagnóstico & Cirugía abdominal previa, enfermedad diverticular & 92 \\
Masculino & Terapéutico & Polipectomía con asa & 74 \\
Masculino & Diagnóstico & Sin factor de riesgo & 54 \\
Femenino & Diagnóstico & Enfermedad diverticular, cirugía abdominal previa & 79 \\
Femenino & Diagnóstico & Cirugía abdominal previa & 69 \\
Masculino & Terapéutico & Enfermedad diverticular, polipectomía con asa & 80 \\
Masculino & Terapéutico & Polipectomía con asa & 73 \\
Femenino & Terapéutico & Enfermedad diverticular & 66 \\
Femenino & Terapéutico & Tumor sigmoides estenosante & 69 \\
Femenino & Diagnóstico & Sin factor de riesgo & 64 \\
Masculino & Terapéutico & Biopsias múltiples & 26 \\
Femenino & Terapéutico & Enfermedad diverticular, polipectomía con asa & 80 \\
\hline
\end{tabular}

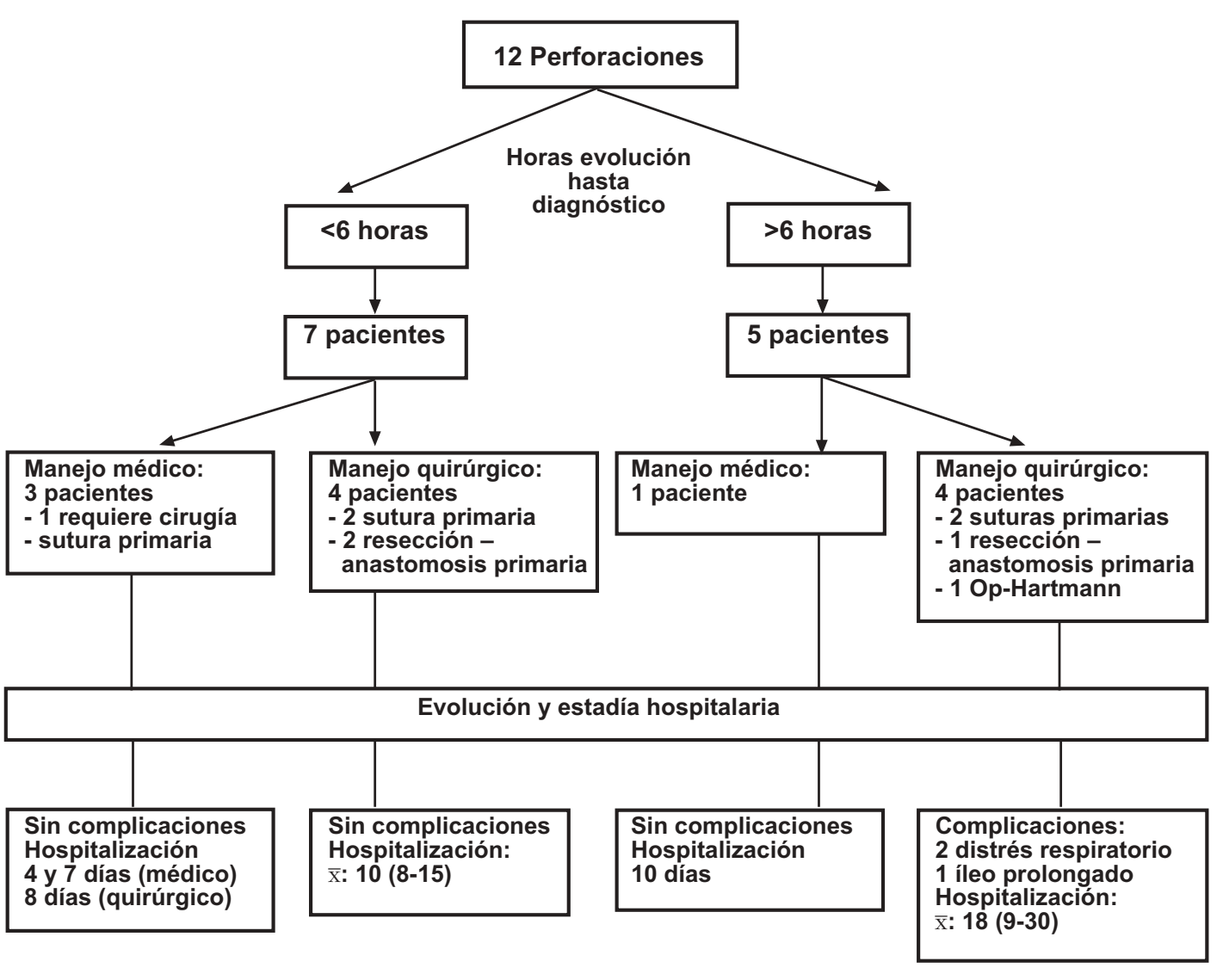

Figura 1. Evolución de los pacientes con perforación colónica. 
Los pacientes con perforación que fueron tratados médicamente permanecieron hospitalizados 4, 7 y 10 días, respectivamente, y los que fueron intervenidos quirúrgicamente estuvieron hospitalizados un promedio de 12 días (r: 8-30).

Al correlacionar la descripción de la colonoscopia con los hallazgos operatorios, se puede inferir que el mecanismo más probable fue la perforación por asa del colonoscopio seguido de la polipectomía con asa de diatermia (Tabla 2).

Complicaciones postoperatorias ocurrieron en 4 pacientes: un paciente presentó un íleo prolongado (mayor a 7 días) que se resolvió en forma espontánea; otro, una atelectasia con sobreinfección pulmonar y, los 2 pacientes más graves, evolucionaron con distrés respiratorio requiriendo ventilación mecánica prolongada. No hubo mortalidad en la serie.

\section{DisCusión}

La perforación de colon en un procedimiento endoscópico es rara, con incidencias comunicadas bajo $0,4 \%$. Esta serie, muestra una incidencia global $(0,1 \%)$ que se encuentra dentro de lo esperado, con una incidencia específica para procedimientos diagnósticos y terapéuticos de $0,056 \%$ y $0,23 \%$, respectivamente, lo que confiere un riesgo 4 veces mayor al terapéutico.

Dentro de los factores comúnmente relacionados a mayor riesgo de perforación, está la pérdida de una adecuada movilidad del colon secundaria a cicatrices abdominales postquirúrgicas, tumores, radioterapia y deformación de colon por enfermedad diverticular ${ }^{12}$. En este estudio encontramos factores de riesgo reconocidos en 9 pacientes y, sólo 2 no presentaron factor alguno.

Los mecanismos que pueden explicar la perforación pueden ser: El barotrauma excesivo, donde el sobreinsuflar el colon provoca pequeñas erosiones lineales en la serosa, que tras la manipulación del endoscopio, resultan en soluciones de continuidad. Por la fuerza mecánica al introducir y manipular el endoscopio contra las paredes del colon, ya sea por el extremo o por la formación del asa. Procedimientos diagnósticos (biopsias) y terapéuticos (cauterio lesiones sangrantes).

En esta serie, el fenómeno que más explicó las perforaciones fue el mecanismo de asa del colonoscopio. Se presentó en procedimientos laboriosos en pacientes con deformidad de colon. Esta situación debe alertar al endoscopista para prevenir manipulaciones excesivas que puedan resultar en lesiones iatrogénicas.

El cuadro debe sospecharse durante el examen si existe visualización directa del mesenterio u órganos abdominales, pérdida de la expansión colónica pese a una adecuada insuflación, distensión abdominal y malestar progresivo del paciente.

La sospecha, una vez terminado el procedimiento, debe considerarse en todo paciente que evoluciona con malestar abdominal tras un procedimiento laborioso o con dolor y distensión

Tabla 2. M ecanismo de perforación supuesto en cada paciente operado

\begin{tabular}{|c|c|c|}
\hline Descripción colonoscopia & Hallazgo operatorio & Mecanismo \\
\hline Ulceras ileales, biopsia borde & Perforaciones puntiformes & Pinza biopsia \\
\hline Colonoscopía normal & Perforación lineal extensa sigmoides & Asa colonoscopio \\
\hline Control cáncer de colon operado & Perforación cicatriz colostomía & Extremo colonoscopio \\
\hline Polipectomía con asa & Perforación parietal redondeada & Polipectomía con asa \\
\hline Enfermedad diverticular masiva & Perforación lineal extensa sigmoides & Asa colonoscopio \\
\hline $\begin{array}{l}\text { Lesión proliferante y estenosante } \\
\text { de sigmoides, biopsias lesión }\end{array}$ & Perforación tumoral contenida & Extremo colonoscopio \\
\hline Colonoscopía normal & Perforación lineal sigmoides & Asa colonoscopio \\
\hline Pólipo colónico, polipectomía & Perforación parietal redondeada & Postpolipectomía \\
\hline $\begin{array}{l}\text { Gran deformidad sigmoidea por } \\
\text { enfermedad diverticular masiva }\end{array}$ & Perforación lineal sigmoides & Asa colonoscopio \\
\hline
\end{tabular}


abdominal persistente. No se encontró en esta revisión ningún procedimiento descrito en el que se reconociera la complicación en forma inmediata, sino más bien fueron pacientes que evolucionaron con disconfort general, distensión y dolor abdominal permanente después del procedimiento. La confirmación diagnóstica se realiza mediante la demostración de aire subdiafragmático, comúnmente a través de una radiografía simple de abdomen de pie. En caso de dudas, la tomografía computada abdominal es el examen de elección para su confirmación. El tratamiento de la perforación es quirúrgico en la mayoría de los casos; no obstante, el tratamiento médico conservador en un grupo seleccionado de pacientes también puede ser exitoso, como muestra la experiencia presentada en numerosas publicaciones internacionales (Tabla 3). Este resulta generalmente exitoso en los casos en que la perforación es pequeña, si ha existido una adecuada preparación de colon y, si desde un principio, el paciente está en buenas condiciones generales y sin signos de irritación peritoneal difusos $^{10-12}$.

El tratamiento médico recomendado consiste en: hospitalización, reposo intestinal (régimen 0), hidratación parenteral y antibióticos endovenosos con cobertura a Gram (-) y anaerobios asociado a una observación clínica permanente y expectante.

El abordaje quirúrgico se plantea sin postergación alguna en los pacientes con signos de irritación peritoneal difusos, malas condiciones generales o la presencia de un tumor de colon concomitante.

Para los pacientes con perforaciones pequeñas y sin contaminación peritoneal masiva, la indicación es la sutura primaria y el aseo peritoneal, lo que ha sido reportado con excelentes resulta$\operatorname{dos}^{13-14}$. Para perforaciones mayores asociadas a daño extenso en la pared del colon sin contaminación peritoneal masiva en un paciente sin sepsis ni compromiso hemodinámico, la resección segmentaria y anastomosis primaria sin ostomía de protección también es segura ${ }^{15-17}$. Para aquellos pacientes con contaminación peritoneal mayor, cuadro séptico en evolución y malas condiciones generales, el aseo peritoneal y la operación de Hartmann sería lo indicado ${ }^{18}$. El abordaje laparoscópico también resulta interesante con buenos resultados en pacientes seleccionados, reduciendo la morbilidad asociada a la laparotomía, con los consabidos beneficios de la cirugía mínimamente invasiva $^{19,20}$.

Se ha publicado recientemente ${ }^{21,22}$ que el uso de clips por vía endoscópica tendría resultados

Tabla 3. Perforaciones de colon en procedimientos endoscópicos y su tratamiento

\begin{tabular}{|c|c|c|c|c|}
\hline Autor & Tiempo (años) & № colonoscopias & № perforaciones & Tratamiento \\
\hline Tulchinsky $^{1}$ & 8 & 12.067 & $7 \quad(0,058 \%)$ & $\begin{array}{ll}6 & \text { quirúrgico } \\
1 & \text { médico }\end{array}$ \\
\hline Araghizadeth $^{3}$ & 30 & 34.620 & $31 \quad(0,09 \%)$ & $\begin{array}{l}20 \text { quirúrgico } \\
11 \text { médico }\end{array}$ \\
\hline Jentschura $^{7}$ & 9 & 29.695 & $31 \quad(0,1 \%)$ & $\begin{aligned} 24 & \text { quirúrgico } \\
7 & \text { médico }\end{aligned}$ \\
\hline Iqbal $^{8}$ & 20 & 135.730 & $111 \quad(0,082 \%)$ & $\begin{aligned} 101 & \text { quirúrgico } \\
10 & \text { médico }\end{aligned}$ \\
\hline Hall $^{11}$ & 6 & 17.500 & $15 \quad(0,09 \%)$ & $\begin{array}{c}14 \text { quirúrgico } \\
1 \text { médico }\end{array}$ \\
\hline Anderson $^{23}$ & 10 & 10.486 & $20 \quad(0,19 \%)$ & $\begin{aligned} 19 & \text { quirúrgico } \\
1 & \text { médico }\end{aligned}$ \\
\hline García & 6 & 11.720 & $12 \quad(0,102 \%)$ & $\begin{array}{l}9 \text { quirúrgico } \\
3 \text { médico }\end{array}$ \\
\hline
\end{tabular}


satisfactorios en modelo animal porcino, lo que podría ser una herramienta terapéutica útil. No obstante, la gran mayoría de las perforaciones de colon son diagnosticadas postprocedimiento, circunstancia en la que el riesgo de realizar una nueva endoscopia y agravar la perforación, contraindica tal procedimiento. La repercusión de la evolución y del costo económico que debe asumir un paciente que presenta una perforación de colon durante una colonoscopia queda claramente evidenciada en estadías hospitalarias prolongadas y procedimien-

\section{REFERENCIAS}

1. Tulchisky H, Madhala-Givon O, Wasserberg N. Incidence and management of colonic perforations: 8 years' experience. World J Gastroenterol 2006; 12: 4211-13.

2. Waye JD, Kahn O, Auerbach ME. Complications of colonoscopy and flexible sigmoidoscopy. Gastrointest Endosc Clin N Am 1996; 6: 343-77.

3. Araghizadeh Fy, Timmcke AE, Opelka FG, Hicks TC, BECK DE. Colonic perforations. Dis Colon Rectum 2001; 44: 713-6.

4. Vernaya AM, Longo WE. Complications of endoscopic polypectomy. Surg Oncol Clin N Am 1996; 5: 663-73.

5. Nivatvongs S. Complications in colonoscopic polypectomy: lessons to learn from an experience with 1576 polyps. Am Surg 1988; 54: 61-3.

6. Korman LY, Overholt BF, Box T, Winker CK. Perforation during colonoscopy in endoscopy ambulatory centers. Gastrointest Endosc 2003; 58 : 554-7.

7. Jentschura D, Raute M, Winter J, Henkel T, Kraus M, Manegolg BC. Complications in endoscopy of the lower gastrointestinal tract. Therapy and prognosis. Surg Endosc 1994; 8: 672-6.

8. Igbal CW, Chun Ys, Farley D. Colonoscopic Perforations: A Retrospective review. J Gastrointest Surg 2005; 9: 1229-36.

9. Orsoni P, Berdah S, Verrier C, Caamaño A, Sastre B, Boutboul R et al. Colonic perforations due to colonoscopy: a retrospective study of 48 cases. Endoscopy 1997; 29: 160-4.

10. Chritie JP, Marrazzo J III. Mini perforation of the colon-not all postpolypectomy perforations re- tos quirúrgicos mayores. Esto debe considerarse para la precisa indicación de todo procedimiento invasivo al momento de discutir con los pacientes, los beneficios y riesgos de éstos.

En resumen, de acuerdo a esta experiencia y las comunicadas previamente en la literatura internacional, podemos decir que la perforación de colon es una complicación poco frecuente, cuyo tratamiento es en general quirúrgico, pero el tratamiento médico puede ser una alternativa segura en casos seleccionados.

quire laparotomy. Dis Colon Rectum 1991; 34: 132-5.

11. Hall C, Dorricott NJ, Donovan IA, Neoptolemos JP. Colon perforation during colonoscopy: Surgical versus Conservative management. Br J Surg 1991; 78: 542-4.

12. MaIRA J. Complicaciones relacionadas con el procedimiento endoscópico. Gastr Latinoam 2004; 15: 137-41.

13. Chappicus CW, Frey DJ, Dietzen CD, Panetta TP, Buechter KL, Cohn I. Management of penetrating colon injuries: a prospective randomized trial. Ann Surg 1991; 213: 492-3.

14. González RP, Merlotti GJ, Holevar MR. Colostomy in penetrating colon injury; is it necessary? J Trauma 1996; 41: 271-5.

15. Kawendo NY, Modiba MC, Matlala NS, Becker PJ. Randomized trial to determine if delay from time of penetrating injury precludes primary repair. $\mathrm{Br}$ J Surg 2002; 89: 993-8.

16. Pascuale M, Fabian TC. Practice management guidelines for trauma from the Eastern Association for the Surgery of Trauma. J Trauma 1998; 44: 941-57.

17. Gedebou TM, Wong RA, Rappaport WD, Jaffe P, Kahsai D, Hunter GC. Clinical presentation and management of iatrogenic colon perforation. Am J Surg 1996; 172: 454-7; discussion 457-8.

18. Adedoyin A, Ekanem E. A ten years study of penetrating injuries of the colon. Dis Colon Rectum 2004; 47: 2169-77.

19. Wulltein CH, Köppen M-O, Gross E. Laparoscopic treatment of colonic perforations related to colonoscopy. Surg Endosc 1999; 13: 484-7.

20. Putcha R, Burdick J. Management of iatrogenic 
perforation. Gastroenterol Clin North Am 2003; 32: 1289-309

21. Raju G, Ahmed I, Brining D, Xiao S. Endoluminal closure of large perforations of colon with clips in a porcine model. Gastrointes Endosc 2006; 64: 640-6.

22. Raju G, Ahmed I, Shubukawa G, Poussard A,
BRINING D. Endoluminal suturing may overcome the limitations of clip closure of a gaping wide colon perforation. Gastrointest Endosc 2007; 65: 503-9.

23. Anderson ML, Pasha TM, Leighton JA. Endoscopic perforation of the colon from a 10 years study. Am J Gastroenterol 2000; 95: 3418-22. 